and pathogenesis of pain and, second, to derive from the various disciplines a "total and unitary concept of pain".

Whilst the first of these aims was realized, the second, regrettably, was not, and at the present stage of knowledge it seems a rather ambitious aim. It is a goal always to be kept in mind, however, and one which will be reached, if ever, by diverse paths. Many of these paths wero described by the contributors to this symposium and they ranged from a study of tail-flick in the rat in response to radiant-heat before and after various analgesics to the perception of pain in man inflicted by electrically stimulating the teeth.

An interesting and intermediate path between the study of rodents and the study of man is represented by the paper in which D. Römer describes analgesic effects in the monkey. The "painful" stimulus was an alternating current passed through the tail, and the monkey's responses included mydriases, slight facial movements with or without crying, baring of teeth with loud crying, and pronounced agitation. Some excellent photographs accompany this paper.

In their preface the editors claim that this volume is unique for the amount of information it contains on all aspects of pain. Its scope can be seen from the titles of its six sections: "Neurophysiological and Psychophysiological Bases of Pain", "Experimental Methods for" Pain and Analgesia", "Biochemical Basis of Analgesia", "Psychopharmacology of Analgesics", "Electrophysiological and Electroencephalographical Bases", and "Clinical and Therapeutical Application".

Although some of the papers were given in Fronch, all except the general conclusions have been translated into English. Unfortunately, some of the translations are far from good and thus render comprehension tiresome and difficult. The "discussions", which appear in the table of contents as a part of each section, do not conform to the usual type of discussion at such symposia but consist rather of a further series of papers.

In his summing-up Professor Soulairac says that in spite of the various approaches to the study of pain there is fairly general agreement on the importance of "levels of vigilance" in the perception of pain and its integration, and it is this concept which has provided perhaps the most interesting basis for research in the pharmacology of pain. He goes on to say that whatever the beliefs of research workers, the criterion of efficiency remains the same; namely, to understand the mechanisms of pain in order to suppress it; it is this which explains the importance of pharmacological and psychopharmacological studies in this field. He ends on a gloomy note saying that the diversity of explanatory hypotheses of pain and analgesia serves only to underline the paucity of present knowledge.

The contributors to this volume ropresent many disciplines and it will therefore be of interest to a wide variety of pcople including neurophysiologists, pyschopharmacologists, psychologists, psychiatrists, anaesthesiologists and neurosurgeons. Diana R. HaslaM

\section{NERVOUS TISSUE GROWTH}

\section{Growth of the Nervous System}

Edited by G. E. W. Wolstenholm and Maeve O'Connor. (A Ciba Foundation Symposium.) Pp. xi + 295. (Churchill: London, 1968.) $65 s$.

This book, the product of a symposium held in June 1967 in London, serves to take stock of progress in experimentation and ideas in the subject. This, together with several imaginative looks into the future, makes for a very interesting and exciting volume. The contributors have sought to investigate various aspects of nervous tissue growth by use of a variety of techniques and experimental material. The benefits gained by these differences in approach revealed themselves particularly well during the spirited discussions, which have apparently been reported in full.

The symposium itself was divided into four main areas: development of specific neuronal connexions; development of movement; role of chemically specific signals in the development of the nervous system; and trophic interaction, peripheral and central. The introductory survey by Szentagothai enables the reader newly interested in this subject to appreciate the problems and the progress made in the past twenty years, and how the approaches have changed with the introduction of new techniques such as the electron microscope. unit level physiology, and radioisotope labelling. They have proved to be especially productive when used in conjunction with each other, and on simple physiological systems.

Briefly, the results reported range from seemingly random synaptic connexions formed in nervous tissue culture experiments, to the specific retino-rectal connexions achieved under various adverse circumstances in the amphibian eye; and from the positive effects of nerve growth factor on neuronal development to the action of cholinergic transmission blockers in their ability to mimic the muscle degeneration produced by surgical section of the nerve. Some important observations are made regarding the dynamic relationship between the axon and the myelin sheath, and the ability of the latter to allow "external" biochemical communication with the axoplasm all along its length. Also included is an investigation of axoplasmic flow in snail and frog CNS/nerve/muscle preparations - which shows a relatively rapid flow ( $1 \mathrm{~cm} / 20 \mathrm{~min}$ in snail nerve), with a consequent release of glutamate at the muscle.

To the non-specialist, some of the papers may prove to be rather hard going, but, having persevered, the rewards are generously measured in terms of stimulated interest and a new appreciation of how the fascinating subject of growth in the nervous system is developing. In the words of the chairman of the symposium, Sir John Eccles: "We have to realize the immensity of the problems as we try to imagine them from our present still primitive viewpoint. Before we knew anything about the problems, these were all solved for us in some magical way by 'mysterine' or what you will". Maybe "mysterine" will suffer exposure in the not too distant future !

The presentation of the book is of a high standard. In particular, the high quality of the electron micrograph reproductions deserves mention. It is essential reading for any who wish to keep abreast of events in the ever widening horizons of neurophysiology.

David R. Garnner

\section{GLOBULIN DISORDERS}

Monoclonal and Polyclonal Hypergammaglobulinemia Clinical and Biological Significance. By Jan Gösta Waldenström. Pp. viii +223. (Cambridge University Press: London, 1968.) $55 s$.

Tне $\gamma$-globulin fraction of serum is associated with antibody activity and its concentration is frequently increased in clinical disorders. Electrophoretic analysis showed that such hypergammaglobulinaemias are divisible into two distinct groups. In one, referred to as monoclonal hypergammaglobulinaemia, the increase is caused by a structurally homogeneous $\gamma$-globulin apparently corresponding to a single species of antibody molecule and arising by proliferation of a single line of cells. In the more common polyclonal hypergammaglobulinaemia as occurs, for example, in chronic infections, there is a generalized increase in $\gamma$-globulin produced by a great diversity of cell lines.

In this book Professor Waldenstrom gives an account 